\title{
2-tert-butyl-primaquine exhibit potent blood schizontocidal antimalarial activity via inhibition of heme crystallization
}

\author{
Nhien Nguyen Thanh Thuy ${ }^{1,2^{*}}$, Huy Nguyen Tien ${ }^{2,3}$, Rahul Jain ${ }^{4}$, Kaeko Kamei ${ }^{2}$ \\ From Parasite to Prevention: Advances in the understanding of malaria \\ Edinburgh, UK. 20-22 October 2010
}

\section{Background}

Primaquine $(\mathrm{PQ})$ is the only 8-quinolinamine available to treat the malarial parasites in the infections caused by Plasmodium vivax and $P$. ovale. PQ has broad range of antimalarial activities, including efficacy as a causal prophylactic, gametocytocide, and sporontocide. These encouraging pharmacological properties make PQ an ideal drug to emulate while designing new antimalarials with improved activities ([1]). The placement of a metabolically stable tert-butyl group at the $\mathrm{C}-2$ position of a quinoline ring in $\mathrm{PQ}$ (2-tert-Butyl-Primaquine - BPQ) results in a tremendous improvement in blood schizontocidal antimalarial activity $([1,2])$. Because free heme released from hemoglobin catabolism in a malarial parasite is highly toxic, the parasite protects itself mainly by crystallization of heme into insoluble nontoxic hemozoin ([3]). In this study, we investigate the mechanism of blood schizontocidal activity of BPQ.

\section{Results}

The ability of 2-tert-butylprimaquine to inhibit in vitro beta-hematin formation (see Table 1), to form a

Table 1 IC50 values for inhibition of $P$. falciparum growth and heme crystallization

\begin{tabular}{lll}
\hline Drug & IC50 $(\boldsymbol{\mu M})$ for inhibition of \\
\cline { 2 - 3 } & P. falciparumD6 clone growth & BH formation \\
\hline $\mathrm{CQ}$ & 0.3 & 15.4 \\
$\mathrm{PQ}$ & $\mathrm{ND}^{*}$ & $\mathrm{ND}^{*}$ \\
$\mathrm{BPQ}$ & 0.1 & 2.9 \\
\hline
\end{tabular}

*ND, not detected.

${ }^{1}$ Oxford University Clinical Research Unit, Hospital for Tropical Diseases, Ho Chi Minh City, Vietnam complex with heme with a stoichiometry of 1:1 (see Figure 1 and Figure 2), and to enhance heme-induced hemolysis (see Figure 3) were demonstrated.

\section{Conclusion}

The results described herein indicate that a major improvement in the blood-schizontocidal antimalarial activity of 2-tert-butylprimaquine might be due to a disturbance of heme catabolism pathway in the malarial parasite.

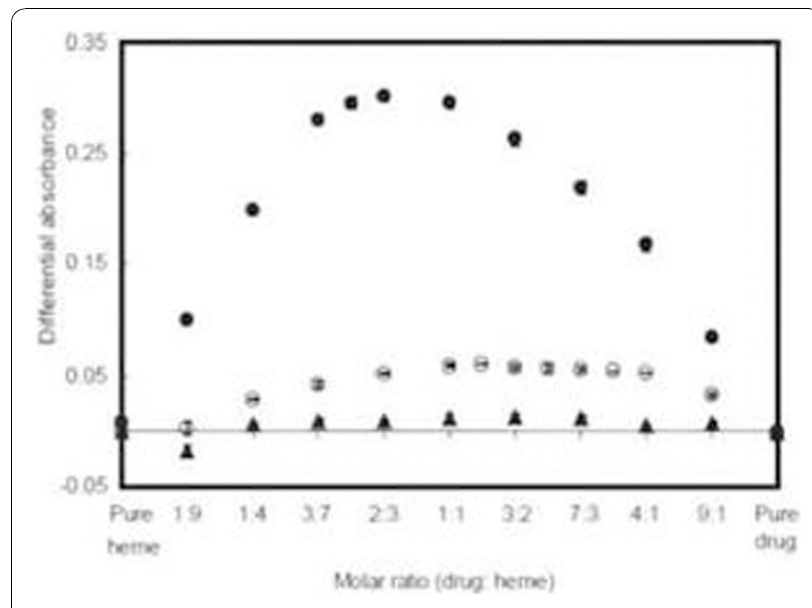

Figure 1 (abstract P69). Job plots of heme-CQ (closed circles), heme-PQ (closed triangles), and heme-BPQ (open circles) interaction. The total final combined concentration of heme and drug in the mixtures was constant at $10 \mu \mathrm{M}$ in $40 \%$ aqueous DMSO. The $\mathrm{pH}$ and the temperature were constant at $\mathrm{pH} 7.4$ and $25^{\circ} \mathrm{C}$. The differential absorbance at $400 \mathrm{~nm}$ was recorded after incubation for $30 \mathrm{~min}$. Values are the means \pm standard errors of the means of three independent experiments. The results are reproducible. 


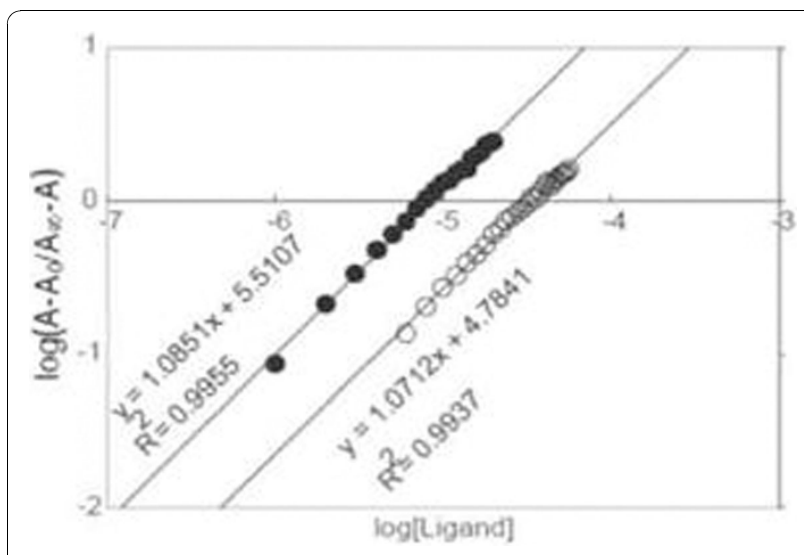

Figure 2 (abstract P69). Hill plots of heme-CQ (closed circles) and heme-BPQ (open circles) association. The $n$ values correspond to individual slopes. The $n$ and $K a$ values for heme-CQ association were 1.09 and $3.24 \times 10^{5} \mathrm{M}^{-1}$, respectively. The $n$ and $K a$ values for heme-BPQ association were 1.07 and $0.61 \times 10^{5} \mathrm{M}^{-1}$, respectively.

\section{Acknowledgements}

Our sincere thanks to all members in Structural Biotechnology Laboratory, Department of Applied Biology, Kyoto Institute of Technology who have contributed to and worked on this study.

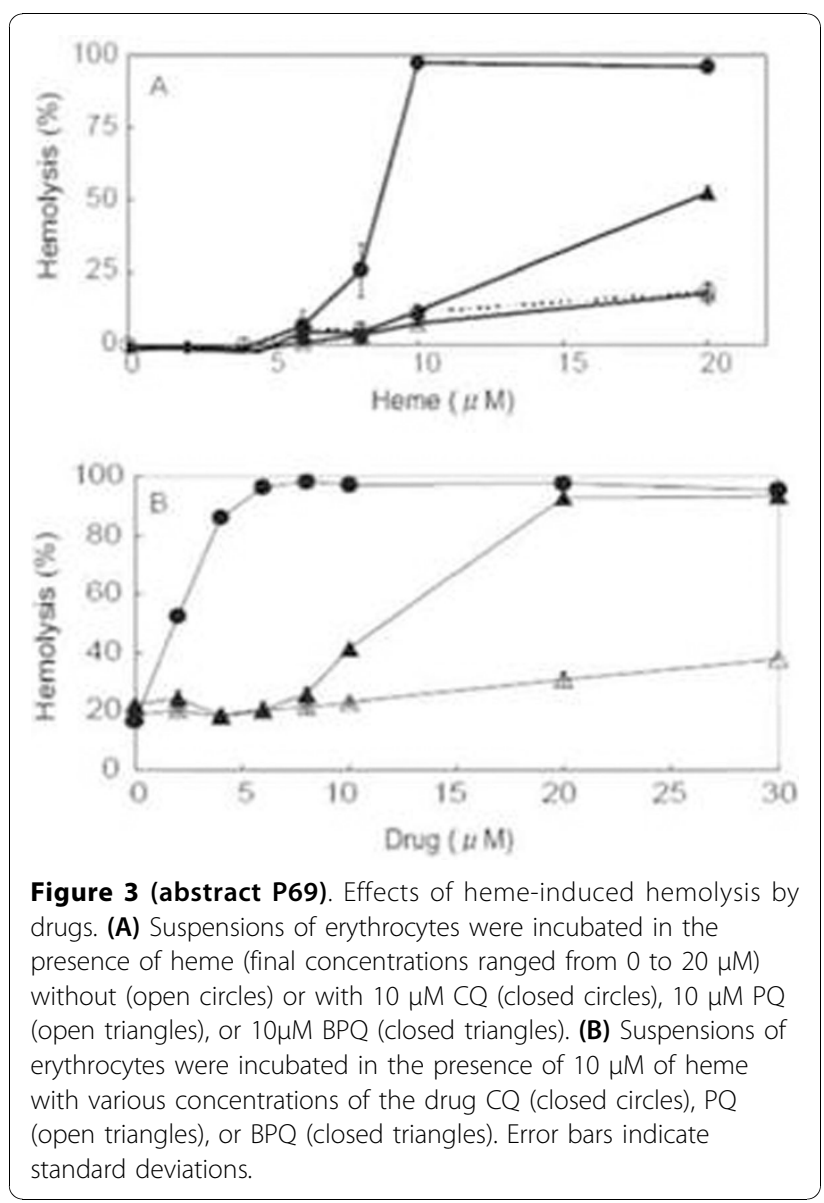

\section{Author details}

'Oxford University Clinical Research Unit, Hospital for Tropical Diseases, Ho Chi Minh City, Vietnam. ${ }^{2}$ Department of Applied Biology, Kyoto Institute of Technology, Kyoto 606-8585, Japan. ${ }^{3}$ Department of Immunogenetics, Institute of Tropical Medicine (NEKKEN), Nagasaki University; Nagasaki 852 8523, Japan. ${ }^{4}$ Department of Medicinal Chemistry, National Institute of Pharmaceutical Education and Research, Sector 67, S.A.S. Nagar, Punjab 160 062, India.

Published: 16 December 2010

\section{References}

1. Jain M, Vangapandu S, Sachdeva S, Singh S, Singh PP, Jena GB, Tikoo K, Ramarao P, Kaul CL, Jain R: Discovery of a bulky 2-tert-butyl group containing primaquine analogue that exhibits potent bloodschizontocidal antimalarial activities and complete elimination of methemoglobin toxicity. J Med Chem 2004, 47:285-287.

2. Jain M, Vangapandu S, Sachdeva S, Jain R: Synthesis and bloodschizontocidal antimalarial activities of 2-substituted/2,5-disubstituted-8quinolinamines and some of their amino acid conjugates. Bioorg Med Chem 2004, 12:1003-1010.

3. Egan TJ, Combrinck JM, Egan J, Hearne GR, Marques HM, Ntenteni S, Sewell BT, Smith PJ, Taylor D, van Schalkwyk DA, Walden JC: Fate of haem iron in the malaria parasite Plasmodium falciparum. Biochem J 2002, 365:343-347.

\section{doi:10.1186/1475-2875-9-S2-P69}

Cite this article as: Thanh Thuy et al: 2-tert-butyl-primaquine exhibit potent blood schizontocidal antimalarial activity via inhibition of heme crystallization. Malaria Journal 2010 9(Suppl 2):P69.

\section{Submit your next manuscript to BioMed Central and take full advantage of:}

- Convenient online submission

- Thorough peer review

- No space constraints or color figure charges

- Immediate publication on acceptance

- Inclusion in PubMed, CAS, Scopus and Google Scholar

- Research which is freely available for redistribution

Submit your manuscript at www.biomedcentral.com/submit 